\title{
Lifetime of a massive particle in a de Sitter universe
}

\author{
Jacques Bros ${ }^{1}$, Henri Epstein ${ }^{2}$ and Ugo Moschella ${ }^{3}$ \\ ${ }^{1}$ Service de Physique théorique - CEA. Saclay. 91191 Gif-sur Yvette. \\ ${ }^{2}$ Institut des Hautes Études Scientifiques, 91440 Bures-sur-Yvette. \\ ${ }^{3}$ Università dell'Insubria, Como and INFN Milano
}

\begin{abstract}
We study particle decay in de Sitter space-time as given by first order perturbation theory in an interacting quantum field theory. We show that for fields with masses above a critical mass $m_{c}$ there is no such thing as particle stability, so that decays forbidden in flat space-time do occur there. The lifetime of such a particle also turns out to be independent of its velocity when that lifetime is comparable with de Sitter radius. Particles with lower mass are even stranger: The masses of their decay products must obey quantification rules, and their lifetime is zero.
\end{abstract}

Some important progress in the astronomical observations of the last ten years [1, 2] have led to the surprising conclusion that the recent universe is dominated by a "dark" exotic form of energy density that acts repulsively at large scales. The simplest and best known candidate for the "dark energy" is the cosmological constant, and the de Sitter geometry, which is the homogeneous and isotropic solution of the cosmological Einstein equations in vacuo, appears to take the double role of reference geometry of the universe, namely the geometry of spacetime deprived of its matter and radiation content and of the geometry that the universe will approach asymptotically.

One might think that the presence of a cosmological constant, while having a huge impact on our understanding of the universe as a whole, would not influence microphysics in its quantum aspects. This is also the viewpoint taken in the context of inflationary models [3], where the effective cosmological constant is many orders of magnitude larger than the one observed today. However this conclusion may have to be reassessed. Indeed, in presence of a cosmological constant, however small, it is the notion of elementary particle itself which has to be reconsidered, since the usual asymptotic theory is based on concepts which refer closely to Minkowski spacetime and to its Fourier representation, and do not apply to the de Sitter universe which is not asymptotically flat; in fact a true asymptotic theory does not exist at present for de Sitter space. A possible basic approach is perturbation theory; unfortunately, calculations of perturbative amplitudes which in the Minkowskian case would be simple or even trivial become rapidly prohibitive or impossible in the de Sitter case: this in spite of the fact that one is dealing with a maximally symmetric manifold.

In this letter we have tackled one such calculation, namely that of the mean lifetime of $\mathrm{dS}$ unstable scalar particles. The results exhibit significant differences compared to the Minkowski case and decay processes which are normally forbidden become possible (as exhibited by Nachtmann [4] in a special case) and vice versa, processes that are normally possible are now forbidden. The maximal symmetry of the dS universe allows for the introduction of a global mass operator, one of the two Casimir operators of the dS group $S O(1, d)$ (see e.g. [5]); this quantity is conserved for dS invariant field theories and it still makes sense to follow Wigner [7] in associating a particle with a unitary irreducible representation of the dS group labeled by a mass parameter, as we do here. However, in contrast with the Poincaré group, the tensor product of two unitary irreducible representations of masses $m_{1}$ and $m_{2}$ decomposes into a direct integral of representations whose masses $m$ do not satisfy the "subadditivity condition" $m \geq m_{1}+m_{2}$ : all representations of mass larger than a certain critical value (principal series) appear in the decomposition. This fact was shown in 6] for the two-dimensional case and will be established here in general. This means that the de Sitter symmetry does not prevent a particle with mass in the principal series from decaying into e.g. pairs of heavier particles. This phenomenon also implies that there can be nothing like a mass gap in that range. This is a major obstruction to attempts at constructing a de Sitter S-matrix; the Minkowskian asymptotic theory makes essential use of an isolated point in the spectrum of the mass operator, and this will generally not occur in the de Sitter case. We will also show that the tensor product of two representations of sufficiently small mass below the critical value (complementary series) contains an additional finite sum of discrete terms in the complementary series itself (at most one term in dimension four). This implies a form of particle stability, but the new phenomenon is that a particle of this kind cannot disintegrate unless the masses of the decay products have certain quantized values. Stability for the same range of masses has also been recently found [8] in a completely different context.

We will resort to first order perturbation theory in our calculations. These are made trivial in the Minkowski case by the use of momentum-space, but this is not so in the de Sitter space. We restrict at first our attention to the principal series and start by deriving a new general formula expressing the decay probability of a particle which applies to both Minkowski and de Sitter spacetimes in dimension $d$, where the de Sitter manifold is identified with the hyperboloid $\left\{x \in \mathbf{R}^{d+1}: x^{2}=\right.$ $\left.x_{0}^{2}-x_{1}^{2}-\ldots-x_{d}^{2}=-R^{2}\right\}$ in the $(d+1)$-dimensional Minkowski space. Differences will come in later.

A Klein-Gordon neutral scalar field $\phi$ with mass $m \geq 0$ is characterized by its two-point vacuum expectation value $w_{m}(x, y)=(\Omega, \phi(x) \phi(y) \Omega)$ which is uniquely 
specified up to a constant factor by requiring invariance, locality, and a suitable spectral condition (having a thermodynamical physical interpretation in the dS case $[9,[10]) . \quad w_{m}$ allows for the reconstruction of the Fock space of the theory and of a representation of the invariance group whose restriction to the one-particle subspace is irreducible and labeled by $m$. In the dS case $m$ can be related to a dimensionless parameter $\nu$ as follows

$$
m^{2} R^{2}=\left(\frac{d-1}{2}\right)^{2}+\nu^{2}
$$

The range $m \geq m_{c}=(d-1) / 2 R$ (i.e. $\nu$ real) corresponds to the principal series while $0 \leq m<m_{c}$ corresponds to the complementary series ( $\nu$ imaginary). These restrictions ensure that $w_{m}$ is positive definite and therefore a quantum theoretical interpretation is available. Consider now an interaction

$$
\int \gamma g(x) \mathcal{L}(x) d x, \quad \mathcal{L}(x)=: \phi_{0}(x) \phi_{1}(x)^{q_{1}} \ldots \phi_{N}(x)^{q_{N}}:
$$

between $1+N$ independent scalar fields $\phi_{0}, \phi_{1}, \ldots, \phi_{N}$ with masses $m_{0}, m_{1}, \ldots, m_{N}$; self-interactions $\mathcal{L}(x)=$ : $\phi_{0}(x)^{n}$ : are a special case of this coupling. The spacetime dependent "switching-on factor" $g(x)$ is there to take care of the infrared divergence of the integrals and amounts to putting the system in a box and allowing for a finite time duration of the interaction. In the end however $g$ should be made to tend to 1 everywhere (adiabatic limit).

Let $\Psi=\int d x f(x) \phi_{0}(x) \Omega$ be a (normalized) oneparticle state created by $\phi_{0}$ from the vacuum; $f(x)$ encodes the physical details about the quantum state of the unstable particle whose disintegration we aim to study. At first order in perturbation theory Wick's theorem gives the transition probability $\Gamma_{1_{0} ; q_{1}, \ldots, q_{N}}$ from $\Psi$ to any possible state containing $q_{1}+q_{2}+\ldots+q_{N}$ particles created respectively by the fields $\phi_{1}, \phi_{2}, \ldots \phi_{N}$ :

$$
\begin{aligned}
& \Gamma=\gamma^{2} \int d x d u d v d y \overline{f(x)} f(y) g(u) g(v) K(x, u, v, y),(2) \\
& K(x, u, v, y)=w_{m_{0}}(x, u) \prod_{j=1}^{N} q_{j} ! w_{m_{j}}(u, v)^{q_{j}} w_{m_{0}}(v, y) .
\end{aligned}
$$

The four-point kernel $K$ admits an easy graphical interpretation in $x$-space as a bubble with two external legs. The product of two-point functions (the bubble) may be replaced by its Källén-Lehmann type representation:

$$
\prod_{j=1}^{N} w_{m_{j}}(u, v)^{q_{j}}=\int d a^{2} \rho\left(a^{2} ; m_{1}, \ldots, m_{n}\right) w_{a}(u, v) .
$$

It is far from obvious that such representation exists; besides, calculating $\rho$ is easy only in the Minkowski case for $n=2$ (see below); difficult or impossible in other cases. While the amplitude (2) is infrared divergent in the limit $g \rightarrow 1$, it is possible to replace just one of the $g$ 's by 1 in the integral. Then one integration can be done by means of the projector identity ( $d y$ denotes either the Poincaré or the dS invariant measure):

$$
\int w_{m}(x, y) w_{m^{\prime}}(y, z) d y=C(m) \delta\left(m^{2}-m^{\prime 2}\right) w_{m}(x, z)
$$

Here the global structure of either the Minkowski or the dS spacetime enters crucially. For dS theories this identity holds only for the principal series; $C(m)=2 \pi$ (Minkowski); $C(\nu)=2 \pi|\operatorname{coth}(\pi \nu)|(\mathrm{dS})$. There follows a general formula for the transition probability:

$$
\begin{aligned}
\Gamma_{1_{0} ; q_{1}, \ldots, q_{N}} & =\frac{\gamma^{2} C\left(m_{0}\right) \int g(x)|F(x)|^{2} d x}{\int \overline{f(x)} w_{m_{0}}(x, y) f(y) d x d y} \\
& \times\left(\prod_{j=1}^{N} q_{j} !\right) \rho\left(m_{0}^{2} ; m_{1}, \ldots, m_{N}\right) .
\end{aligned}
$$

Here $F(x)=\int w_{m_{0}}(x, y) f(y) d y$ is the wavefunction associated to $f$; the denominator is the squared norm of $\Psi$ no longer assumed to be one. This formula has an interesting simple structure: the first factor does not depend on the number or nature of the decay particles but only on the wavefunction of the incoming unstable particle. The infrared problem is contained in this factor and has to be overcome when letting the remaining $g(x)$ tend to 1 . The second factor is just the relevant KällénLehmann weight times the right combinatorial factor.

We now focus on the decay of a particle of mass $m_{0}$ into two particles of mass $m_{1}$ and briefly discuss the wellknown Minkowski case first. The weight $\rho\left(m_{0}^{2} ; m_{1}, m_{1}\right)$ can be computed by Fourier transforming $w_{m_{1}}^{2}(x, y)$ :

$$
\rho=\frac{\left(m_{0}^{2}-4 m_{1}^{2}\right)^{\frac{d-3}{2}}}{(4 \pi)^{\frac{d-3}{2}} 2^{d} \pi m_{0} \Gamma\left(\frac{d-1}{2}\right)} \theta\left(m_{0}^{2}-4 m_{1}^{2}\right) .
$$

The appearance of Heaviside's $\theta$ function forbids the decay of a particle into two that are globally heavier. This is a familiar consequence of the Poincaré invariance of the theory. As for the adiabatic limit, the common choice is to choose $g(x)$ as the characteristic function of some time interval $T$. It is then found that the transition probability (4) is proportional to $T$ and thus diverges when $T \rightarrow \infty$. Fermi's golden rule tells us that the transition probability per unit time (see e.g. [11]) has a finite limit

$$
\begin{aligned}
\frac{1}{\tau}= & \lim _{T \rightarrow \infty} \frac{\Gamma_{1_{0} ; 2_{1}}}{T}=2 \rho\left(m_{0}^{2} ; m_{1}, m_{1}\right) \times \\
& \frac{(2 \pi) \gamma^{2} \int\left(2 p^{0}\right)^{-1}|\tilde{f}(p)|^{2} \delta\left(p^{2}-m_{0}^{2}\right) \theta\left(p^{0}\right) d p}{\int|\widetilde{f}(p)|^{2} \delta\left(p^{2}-m_{0}^{2}\right) \theta\left(p^{0}\right) d p}
\end{aligned}
$$

$\tilde{f}(p)$ is the Fourier transform of the wavepacket $f(x)$. The crucial factor $\left(2 p^{0}\right)^{-1}$ in the numerator controls the dependence of the result (6) on the wavepacket $f$. In particular, the decay rate of a particle at rest in our frame can be obtained by letting $|\widetilde{f}(p)|^{2}$ tend to $\delta(\vec{p})$. In this 
limit the factor $\left(2 p^{0}\right)^{-1}$ becomes $\left(2 m_{0}\right)^{-1}$ and one gets:

$$
\frac{1}{\tau_{0}}=\frac{2^{1-d} \gamma^{2}}{\Gamma\left(\frac{d-1}{2}\right)} \frac{1}{m_{0}^{2}}\left(\frac{m_{0}^{2}-4 m_{1}^{2}}{4 \pi}\right)^{\frac{d-3}{2}} \theta\left(m_{0}^{2}-4 m_{1}^{2}\right) .
$$

Had we chosen the wavepacket of a particle with sharp momentum $\vec{p}$, we would have obtained an extra Lorentz factor and the lifetime would be longer:

$$
\tau(\vec{v})=\tau_{0}\left(1-v^{2} / c^{2}\right)^{-\frac{1}{2}}, \quad \vec{v}=c \vec{p} / p^{0} .
$$

In the de Sitter case we use the dimensionless parameter $\nu$ (see Eq. 1) to label the two-point functions; they are proportional to Legendre functions of the first kind:

$$
\begin{aligned}
& w_{\nu}\left(z, z^{\prime}\right)=w_{\nu}(\zeta)=\frac{\Gamma\left(\frac{d-1}{2}+i \nu\right) \Gamma\left(\frac{d-1}{2}-i \nu\right)}{2(2 \pi)^{\frac{d}{2}} R^{d-2}} \times \\
& \times\left(\zeta^{2}-1\right)^{-\frac{d-2}{4}} P_{-\frac{1}{2}+i \nu}^{-\frac{d-2}{2}}(\zeta) ;
\end{aligned}
$$

$z, z^{\prime}$ belong to suitable tubular domains of the complex de Sitter spacetime; $\zeta=z \cdot z^{\prime} / R^{2}$ in the ambient spacetime sense (see 9] for details). For $d=2$ the positivity of $\rho\left(\nu^{2} ; \nu, \ldots, \nu\right)$ has already been established [4] for the disintegration into an odd number of particles having the same mass as the unstable particle. Here we need to actually compute the Källén-Lehmann weight i.e. to obtain $\rho\left(\kappa^{2} ; \nu, \nu\right) \equiv \rho_{\nu}(\kappa)$ such that

$$
w_{\nu}^{2}(\zeta)=\int_{0}^{\infty} d \kappa^{2} \rho_{\nu}(\kappa) w_{\kappa}(\zeta)=\int_{-\infty}^{\infty} \kappa d \kappa \rho_{\nu}(\kappa) w_{\kappa}(\zeta) .
$$

Due to (9), the generalized Mehler-Fock theorem [12] tells us that $\rho_{\nu}(\kappa)$ can be expressed as the integral

$$
\begin{aligned}
& \rho_{\nu}(\kappa)=\frac{\left(\Gamma\left(\frac{d-1}{2}+i \nu\right) \Gamma\left(\frac{d-1}{2}-i \nu\right)\right)^{2} \sinh \pi \kappa}{2(2 \pi)^{1+\frac{d}{2}} R^{d-2}} \times \\
& \times \int_{1}^{\infty} P_{-\frac{1}{2}+i \kappa}^{-\frac{d-2}{2}}(x)\left[P_{-\frac{1}{2}+i \nu}^{-\frac{d-2}{2}}(x)\right]^{2}\left(x^{2}-1\right)^{-\frac{d-2}{4}} d x
\end{aligned}
$$

which is well defined for masses such that $|\operatorname{Im} \nu|<\frac{d-1}{4}$; this includes the principal series and a portion of the complementary series. Computing (11) involves only trigonometric functions for odd $d$ [13]. Remarkably, Mellin transform techniques [14] allow the computation for any dimension $d$ (details will be published elsewhere):

$$
\begin{aligned}
\rho_{\nu}(\kappa)= & \frac{R^{2-d} \sinh \pi \kappa}{(4 \pi)^{\frac{d+2}{2}} \sqrt{\pi} \Gamma\left(\frac{d-1}{2}\right)} \frac{\Gamma\left(\frac{d-1}{4}+\frac{i \kappa}{2}\right) \Gamma\left(\frac{d-1}{4}-\frac{i \kappa}{2}\right)}{\Gamma\left(\frac{d+1}{4}+\frac{i \kappa}{2}\right) \Gamma\left(\frac{d+1}{4}-\frac{i \kappa}{2}\right)} \\
& \times \prod_{\epsilon, \epsilon^{\prime}= \pm} \Gamma\left(\frac{d-1}{4}+i \epsilon \nu+\frac{i \epsilon^{\prime} \kappa}{2}\right)
\end{aligned}
$$

Contrary to (5), the weight $\rho$ never vanishes. This means that for $m>m_{c}$ decay processes into heavier particles are always possible and thus, in that range of masses, one is not allowed to draw conclusions about the stability of a certain particle just from its being the lightest in a hierarchy. The Minkowskian result (5) is however recovered in the limit of zero curvature $(R \rightarrow \infty)$ that is achieved by setting $\kappa=m_{0} R$ and $\nu=m_{1} R$. Lowest order corrections to the flat case give:

$$
\begin{aligned}
& R^{2} \rho_{m_{1} R}\left(m_{0} R\right) \sim \frac{|\Delta m|^{\frac{d-3}{2}}}{2^{d} \pi^{\frac{d-1}{2}} \Gamma\left(\frac{d-1}{2}\right) m_{0}}\left(\frac{m_{0}+2 m_{1}}{4}\right)^{\frac{d-3}{2}} \times \\
& \quad \times\left(1+\frac{A}{R^{2}}\right)\left[\theta(\Delta m)+e^{-|\Delta m| R} \theta(-\Delta m)\right],
\end{aligned}
$$

$\Delta m=m_{0}-2 m_{1}$. The lack of particle stability $(\Delta m<$ 0 ) is exponentially small in $R$. If $\Delta m>0$ there is a correction to the flat case of the order of the cosmological constant $\Lambda=\frac{(d-1)(d-2)}{2 R^{2}}$. In the four dimensional case

$$
A=\frac{17}{64\left(m_{1}+\frac{m_{0}}{2}\right)^{2}}-\frac{107}{24 m_{0}^{2}}+\frac{17}{64\left(\frac{m_{0}}{2}-m_{1}\right)^{2}}
$$

All these effects are of course extremely small with the current value of the cosmological constant. What about particle physics at inflation? At that epoch $m R \sim$ $m \times 10^{-15} \mathrm{GeV}^{-1} \ll \frac{3}{4}$ for every particle of reasonable mass. Our results should therefore be extended to the remaining portion of the complementary series $\frac{d-1}{4}<|\operatorname{Im} \nu|<\frac{d-1}{2}$ where all scalar particles lie at the inflation era (but: there is no complementary series in the Fermionic case). By analytic continuation of (12) in $\nu$,

$$
\begin{array}{r}
w_{\nu}^{2}=\int_{-\infty}^{\infty} \kappa d \kappa \rho_{\nu}(\kappa) w_{\kappa}+\sum_{n=0}^{N-1} A_{n}(\nu) w_{i(\mu+2 i \nu+2 n)} \\
A_{n}(\nu)=\frac{8 \pi(-1)^{n}}{n ! 2^{d} \pi^{\frac{1+d}{2}} R^{d-2} \Gamma(\mu)} \frac{\Gamma(\mu+2 i \nu+n) \Gamma(-2 i \nu-n)}{\Gamma(\mu+2 i \nu+2 n) \Gamma(-\mu-2 i \nu-2 n)} \\
\times \frac{\Gamma(\mu+n) \Gamma(-i \nu-n) \Gamma(\mu+i \nu+n)}{\Gamma\left(-i \nu-n+\frac{1}{2}\right) \Gamma\left(\mu+i \nu+n+\frac{1}{2}\right)}
\end{array}
$$

where $\mu=(d-1) / 2$. The number of discrete terms is the largest $N$ satisfying $N<1+|\operatorname{Im} \nu|-\mu / 2$, or 0 if this is negative. A particle of the complementary series with parameter $\kappa=i \beta$ can only decay into two particles with parameter $\nu=\frac{i}{2}(|\beta|+\mu+2 n)$, where $n$ is any integer such that $0 \leq 2 n<\mu-|\beta|$, and the decay is instantaneous. A particle with mass $m \ll m_{c}$ can only decay into two particles of mass $m_{1} \sim m / \sqrt{2}$. Even if the geometry of the universe at inflation was not exactly de Sitterian, this example indicates that quantum field theoretical arguments concerning particle physics at inflation might need revision.

In trying to interpret the above results one can wonder whether they might be due to the thermodynamical properties [9, 10] of the fundamental state we have been using. We have tested this possibility against a similar computation in flat thermal field theory that however does not exhibit this phenomenon in two-particle decays. Another issue has to do with energy conservation and the relation mass/energy. dS invariant field theories admit ten conserved quantities (in $d=4$ ). The identification of a conserved energy among these quantities has 
proven to be useful in classical field theory [15]. The same quantity remains exactly conserved also at the quantum level although it becomes an operator whose spectrum is not positive [9] even when restricted to the region where the corresponding classical expression is positive [15]; the thermodynamical properties of dS fields arise precisely in this restriction [9]. Energy is conserved also in the decay processes that violate mass subadditivity, once the adiabatic limit has been performed. The breakdown of the subadditivity property of masses in dS spacetime just reflects the nonexistence of an Abelian translation group and thereby of a linear energy-momentum space.

We now consider the adiabatic limit problem and its meaning in the de Sitter context, in the case when all particles are in the principal series. A first complication is the existence of several choices of cosmic time, having different physical implications and the result might depend on one's preferred choice. In the closed model, the cosmic time $t$ is related to the ambient space coordinates as follows: $x^{0}=R \sinh (t / R)$. In strict analogy to the Minkowski case, $g(x)$ can be chosen as the indicator function of some cosmic time interval $T$, say $g(x)=g_{T}(x)=\theta(T / 2-|t|)$.

In the flat model the situation is a bit more tricky. Cosmic time is now defined by the relation $x^{0}+x^{d}=$ $R \exp (t / R)$; flat coordinates cover only half of the de Sitter manifold, namely all the events such that $x^{0}+x^{d}>$ 0 . If we introduce the characteristic function $h_{T}(x)=$ $\theta\left(R e^{T / 2 R}-x^{0}-x^{d}\right) \theta\left(x^{0}+x^{d}-R e^{-T / 2 R}\right)$ then we have to add the contribution coming from the other half, i.e. $g(x)=g_{T}(x)=h_{T}(x)+h_{T}(-x)$. With these premises we have found that in both models the first factor in (4) diverges like $T$; thus it has to be divided by $T$ to extract a finite result which is the same in both models:

$$
\lim _{T \rightarrow \infty} \frac{\gamma^{2} C(\kappa) \int g(x)|F(x)|^{2} d x}{T \int \overline{f(x)} w_{\kappa}(x, y) f(y) d x d y}=\frac{\gamma^{2} \pi \operatorname{coth}(\pi \kappa)^{2}}{|\kappa|}(15)
$$

Here the second (unforeseen) result comes in: in con- trast to the Minkowskian case the limiting probability per unit of time does not depend on the wavepacket! This result seems to contradict what we see everyday in laboratory experiments, a well known effect of special relativity (Eq. 7). Furthermore, in contrast with the violation of particle stability that is exponentially small in the de Sitter radius, this phenomenon does not depend on how small is the cosmological constant. How can we solve this paradox and reconcile the result with everyday experience? The point is that the idea of probability per unit time (Fermi's golden rule) has no scale-invariant meaning in de Sitter: if we use the limiting probability to evaluate amplitudes of processes that take place in a short time we get a grossly wrong result. This is in strong disagreement with what happens in the Minkowski case where the limiting probability is attained almost immediately (i.e. already for finite $T$ ). Therefore to describe what we are really doing in a laboratory we should not take the limit $T \rightarrow \infty$ and rather use the probability per unit of time relative to a laboratory consistent scale of time. In that case we will recover all the standard wisdom even in presence of a cosmological constant. But, if an unstable particle lives a very long time $(>>R)$ and we can accumulate observations then a nonvanishing cosmological constant would radically modify the Minkowski result and de Sitter invariant result will emerge. This result should not be shocking: after all erasing any inhomogeneity is precisely what the quasi de Sitter phase is supposed to do at the epoch of inflation; in the same way, from the viewpoint of an accelerating universe all the long-lived particles look as if they were at rest and so their lifetime would not depend on their peculiar motion.

We thank T. Damour, M. Gaudin, G. Gibbons, M. Milgram and V. Pasquier for enlightening discussions. U. M. thanks the SPhT and the IHES for hospitality and support.
[1] A. G. Riess et al., Astronomical Journal 116, 1009 (1998).

[2] S. Perlmutter et al., Astrophys.J.517:565 (1999)

[3] A. Guth, Phys. Rev. D23, 347 (1981). A. Linde, "Particle Physics And Inflationary Cosmology," Chur: Harwood (1990).

[4] O. Nachtmann: Sitz. Ber.Ost. Ak. d. Wiss. II 176, 363 (1968).

[5] F. Gürsey, Introduction to the de Sitter group. In: Group theoretical concepts and methods in elementary particle physics pp. 365-389 Gordon and Breach, New York (1964)

[6] Y. A. Verdiyev, "Harmonic Analysis on Homogeneous Spaces", Hadronic Press, (1986).

[7] E. Wigner, Ann. of Math. (2) 40 (1939), no. 1, 149-204.

[8] K. Skenderis and P. K. Townsend, J. Phys. A 40, 6733 (2007).

[9] J. Bros, J. P. Gazeau and U. Moschella, Phys. Rev. Lett.
73 (1994) 1746. J. Bros and U. Moschella, Rev. Math. Phys. 8 (1996) 327. J. Bros, H. Epstein and U. Moschella, Commun. Math. Phys. 196, 535 (1998)

[10] G. W. Gibbons and S. W. Hawking, Phys. Rev. D 15 (1977) 2738.

[11] M. Veltman, "Diagrammatica", Cambridge University Press, (1994).

[12] W. Magnus, F. Oberhettinger, and R. P. Soni, "Formulas and Theorems for the Special Functions of Mathematical Physics", Berlin: Springer-Verlag (1966).

[13] M. Gaudin and V. Pasquier, unpublished

[14] O. I. Marichev: "Handbook of Integral Transforms of Higher Transcendental Functions". Chichester: Horwood, 1982

[15] L. F. Abbott and S. Deser, Nucl. Phys. B 195, 76 (1982). 